\title{
Hyperbaric oxygen promotes malignant glioma cell growth and inhibits cell apoptosis
}

\author{
YONG-GANG WANG ${ }^{1}$, YI-PING ZHAN ${ }^{1}$, SHU-YI PAN ${ }^{2}$, HAI-DONG WANG ${ }^{2}$,

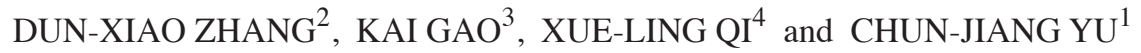 \\ ${ }^{1}$ Department of Neurosurgery, Beijing Sanbo Brain Hospital, Capital Medical University, Beijing 100093; \\ ${ }^{2}$ Department of Hyperbaric Oxygen, Navy General Hospital, Beijing 100048; ${ }^{3}$ Institute of Laboratory Animal Sciences, \\ Peking Union Medical College \& Chinese Academy of Medical Sciences, Beijing 100021; \\ ${ }^{4}$ Department of Pathology, Beijing Sanbo Brain Hospital, Capital Medical University, Beijing 100093, P.R. China
}

Received August 6, 2014; Accepted April 13, 2015

DOI: $10.3892 / \mathrm{ol} .2015 .3244$

\begin{abstract}
Glioblastoma multiforme (GBM) is the most frequently diagnosed intracranial malignant tumor in adults. Clinical studies have indicated that hyperbaric oxygen may improve the prognosis and reduce complications in glioma patients; however, the specific mechanism by which this occurs remains unknown. The present study investigated the direct effects of hyperbaric oxygen stimulation on glioma by constructing an intracranial transplanted glioma model in congenic C57BL/6J mice. Bioluminescent imaging (BLI) was used to assess the growth of intracranial transplanted GL261-Luc glioma cells in vivo, while flow cytometric and immunohistochemical assays were used to detect and compare the expression of the biomarkers, Ki-67, CD34 and TUNEL, reflecting the cell cycle, apoptosis and angiogenesis. BLI demonstrated that hyperbaric oxygen promoted the growth of intracranially transplanted GL261-Luc glioma cells in vivo. Flow cytometric analysis indicated that hyperbaric oxygen promoted GL261-Luc glioma cell proliferation and also prevented cell cycle arrest. In addition, hyperbaric oxygen inhibited the apoptosis of the transplanted glioma cells. Immunohistochemical analysis also indicated that hyperbaric oxygen increased positive staining for Ki-67 and CD34, while reducing staining for TUNEL (a marker of apoptosis). The microvessel density was significantly increased in the hyperbaric oxygen treatment group compared with the control group. In conclusion, hyperbaric oxygen treatment promoted the growth of transplanted malignant glioma cells in vivo and also inhibited the apoptosis of these cells.
\end{abstract}

Correspondence to: Professor Chun-Jiang Yu, Department of Neurosurgery, Beijing Sanbo Brain Hospital, Capital Medical University, 50 Xiangshan Yi-Ke-Song, Beijing 100093, P.R. China E-mail: yucjcmu@163.com

Key words: hyperbaric oxygen, glioma, growth, apoptosis

\section{Introduction}

Gliomas originate from glial cells and are the most common type of intracranial tumor, accounting for 40-60\% of brain tumors $(1,2)$. Glioblastoma multiforme (GBM) is the most frequently diagnosed intracranial malignant tumor in adults (3). The prognosis for GBM patients is invariably poor, even when the patients receive complete surgical resection combined with chemoradiotherapy $(1,4)$. According to a previous study, the postoperative median survival time for GBM patients was $\leq 15$ months, and only $26.5 \%$ of patients survived for 2 years after diagnosis (1).

Hypoxia-induced apoptosis and angiogenesis are key indicators for the diagnosis of GBM. A large hypoxic area in the GBM tissue is associated with poorer prognosis in GBM patients $(5,6)$. Previous studies have indicated that glioblastoma stem cells were present in the hypoxic area and developed resistance to chemotherapy and radiotherapy (7). By contrast, well-differentiated glioblastoma cells were located in the marginal and well-perfused areas of tumor tissues $(8,9)$. Previous studies proposed that hyperbaric oxygen stimulation prior to chemotherapy and radiotherapy may increase the efficacy of the treatment and improve prognosis, as hyperbaric oxygen may increase the oxygen content of tumor tissue $(10,11)$. Hyperbaric oxygen stimulation of GBM patients has previously resulted in longer median survival rates and reduced side effects (12-15). Temozolomide (TMZ) is a novel drug for the treatment of glioma. The cytotoxicity effects of TMZ on chemotherapy-resistant and chemotherapy-sensitive cells increased when the cells were also stimulated with hyperbaric oxygen (16). In vivo, TMZ treatment combined with hyperbaric oxygen may reduce angiogenesis, increase apoptosis and inhibit drug resistance in tumor cells (17).

The direct effect of hyperbaric oxygen stimulation on malignant glioma cells remains unknown. Stuhr et al (18) proposed that hyperbaric oxygen may inhibit the growth of subcutaneous transplanted glioma cells in C57BL/6J mice; however, no cytological experiments were performed and the subcutaneous transplanted gliomas were quite different from intracranial gliomas, which originate from glia cells in situ (19). 
The influence of hyperbaric oxygen on non-tumor cells is complicated: Short exposure to hyperbaric oxygen has been demonstrated to promote tumor cell proliferation, while long exposure results in apoptosis and inhibits proliferation (20). In addition, another previous study demonstrated that hyperbaric oxygen promoted angiogenesis by inducing oxidative stress under physiological conditions (21). In the present study, an intracranial transplanted glioma model in congenic mice was constructed to investigate the direct effects of hyperbaric oxygen stimulation on transplanted glioma cells in vivo.

\section{Materials and methods}

Cell culture. The human glioma GL261/GL261-Luc cell line was obtained from the Division of Cancer Treatment and Diagnosis Tumor Repository, National Cancer Institute (NCI; Frederick, MD, USA). This cell line was routinely cultured in RPMI 1640 medium (GE Healthcare Life Sciences, Logan, UT, USA) supplemented with heat-inactivated fetal bovine serum (Gibco Life Technologies, Carlsbad, CA, USA) in a humidified atmosphere with $5 \% \mathrm{CO}_{2}$ at $37^{\circ} \mathrm{C}$.

Tumor xenograft assay. Inbred 10-week-old female congenic $\mathrm{C} 57 \mathrm{BL} / 6 \mathrm{~J}$ mice (NCI) were maintained under pathogen-free conditions. GL261-Luc glioma cells were injected into the caudate nucleus of the right brain hemisphere, as a $5 \mu 1$ suspension of $5 \times 10^{5}$ cells $\left(1 \times 10^{8}\right.$ cells $\left./ \mathrm{ml}\right)$ in an equal volume of Matrigel (BD Biosciences, Franklin Lakes, NJ, USA). A total of 12 mice were divided into experimental $(n=6)$ and control groups $(n=6)$ and treated accordingly; the two groups were raised in the same conditions, however, the mice in the experimental group were exposed to the hyperbaric intervention process. In vivo bioluminescence imaging (BLI) was applied to measure the tumor volumes. This study was approved by the ethics committee of Beijing Sanbo Brain Hospital (Beijing, China).

Hyperbaric oxygen intervention process. Mice in the hyperbaric oxygen treatment group were placed into an NG90-IIIB medical hyperbaric oxygen chamber (Ningbo Hyperbaric Oxygen Chamber Plant, Ningbo, China) and pressurized to 2.5 atmospheres at a rate of $0.015 \mathrm{MPa} / \mathrm{min}$ for $10 \mathrm{~min}$. This pressure was maintained for $60 \mathrm{~min}$ and decompression was performed at the same rate for $10 \mathrm{~min}$. The hyperbaric oxygen intervention process was performed daily for 10 days.

Immunohistochemical staining. Following 10 days of exposure to the hyperbaric oxygen intervention process, the C57BL/6J mice injected with GL261-Luc glioma cells were sacrificed. Next, the tumors were removed, fixed in $10 \%$ formalin for $24 \mathrm{~h}$, embedded in paraffin and sectioned at $5 \mu \mathrm{m}$ thickness. Following rehydration, antigen retrieval was performed by heating in EDTA ( $\mathrm{pH}$ 8.0) for 15 minutes, and endogenous peroxidase activity was blocked using $3 \%$ hydrogen peroxide at $37^{\circ} \mathrm{C}$ for $10 \mathrm{~min}$. Nonspecific binding was blocked using $3 \%$ skimmed milk for $2 \mathrm{~h}$ at $37^{\circ} \mathrm{C}$. Rabbit anti-human polyclonal Ki-67 (1:500; cat. no. ab15580; Abcam, Cambridge, MA, USA) and rat anti-human monoclonal CD34 (1:100; cat. no. ab8158; Abcam) antibodies and TUNEL reagent (cat. no. ab66108; Abcam) were applied to each slide and incubated at $37^{\circ} \mathrm{C}$ for $2 \mathrm{~h}$. The slides were then incubated using an Envision ${ }^{\mathrm{TM}}$ kit (Dako, Glostrup, Denmark) for 30 min following 3 washes, while 3,3'-diaminobenzidine (Sigma-Aldrich, St. Louis, MO, USA) was used as a chromogen. The sections were counterstained with hematoxylin (Sigma-Aldrich), dehydrated with graded ethanol and xylene and mounted in neutral balsam (CWBio, Inc., Beijing, China).

Flow cytometry. GL261 glioma cells $\left(\sim 1 \times 10^{6}\right)$ were harvested by $0.25 \%$ EDTA and then fixated in pre-cooled $75 \%$ ethanol for $2 \mathrm{~h}$ at $4^{\circ} \mathrm{C}$. Subsequently, the cells were washed twice with phosphate-buffered saline (PBS), resuspended in $500 \mu \mathrm{l}$ PBS with $1 \mu \mathrm{g} / \mathrm{ml}$ RNase A (Life Technologies, Grand Island, $\mathrm{NY}$, USA) and $40 \mu \mathrm{g} / \mathrm{ml}$ propidium iodide (PI; Biotool, Houston, TX, USA), and then incubated at $37^{\circ} \mathrm{C}$ for $30 \mathrm{~min}$. An Annexin V/PI kit (Biotool) was used to detect the externalization of phosphatidylserine in apoptotic cells and all experiments were conducted according to the manufacturer's instructions. The cells were immediately analyzed by flow cytometry (BD Biosciences) with Cell Quest Lysis II software (BD Biosciences), and 5,000 events were observed.

Statistical analysis. The data from at least three independent experiments were analyzed using a two-tailed Student's t-test. The data are presented as the mean \pm standard error (SE), in which the $\mathrm{SE}$ is presented as the error bars. $\mathrm{P}<0.05$ was considered to indicate a statistically significant difference. SPSS software package (version 14.0; SPSS, Inc., Chicago, IL, USA) was used for data analysis.

\section{Results}

Hyperbaric oxygen promoted growth of transplanted glioma. After 10 days of GL261-Luc inoculation, BLI was employed to gain real-time images of the tumors in vivo (Fig. 1A). The photon area and photon sum were also determined using the subsidiary software. No statistically significant difference was identified between the light-emitting area of the hyperbaric oxygen group and the control group (476.67 \pm 190.46 vs. $498.83 \pm 273.73 ; \mathrm{P}=0.872)$. The difference between the sum of emitted photons $(\mathrm{x} 1,000)$ in the two groups was also not statistically significant $(5,677.83 \pm 2,346.48$ vs. $6,069.17 \pm 3,430.37$; $\mathrm{P}=0.822$; Fig. $1 \mathrm{~B}$ and $\mathrm{C}$ ).

To investigate the effects of hyperbaric oxygen on intracranial glioma cells, 6 congenic C57BL/6J mice were subjected to repeated hyperbaric oxygen stimulation at day 10 after inoculation. After a further 10 days from the repeated hyperbaric oxygen treatment, further real-time images were obtained in the same congenic C57BL/6J mice (Fig. 1A). The light-emitting area of the intracranial glioma in the hyperbaric oxygen group was significantly increased compared with the control group $(2,767.50 \pm 811.44$ vs. 1,744.50 \pm 572.04 ; $\mathrm{P}=0.030)$. The sum of emitted photons $(\mathrm{x} 1,000)$ was also significantly increased in the hyperbaric oxygen group compared with the control group $(45,642.50 \pm 17,613.99$ vs. $22,217.83 \pm 7,273.73 ; \mathrm{P}=0.013$; Fig. $1 \mathrm{~B}$ and $\mathrm{C}$ ).

Hyperbaric oxygen promoted glioma cell proliferation and escape from cell cycle arrest. Flow cytometric analysis 
A

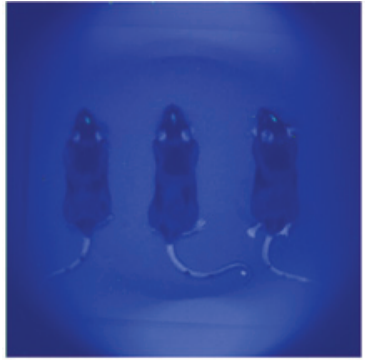

Day 10

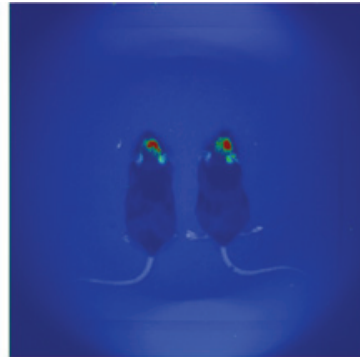

Day 20 HBO

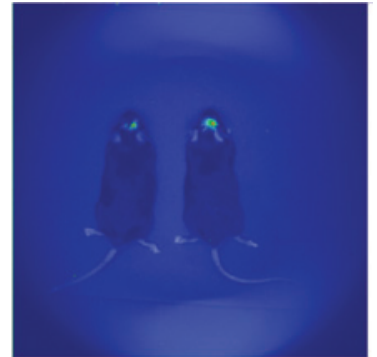

Day 20 control
B

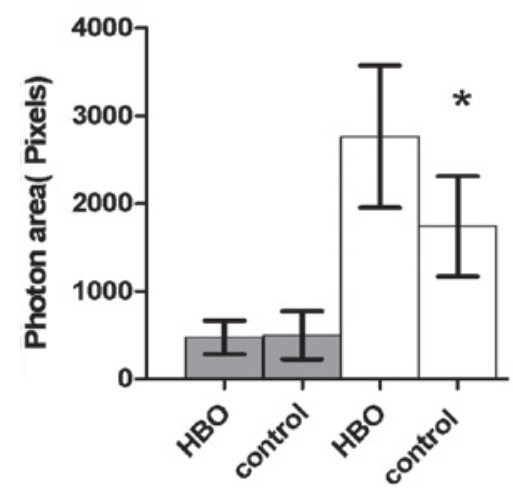

C

C $\quad \times 10^{3}$

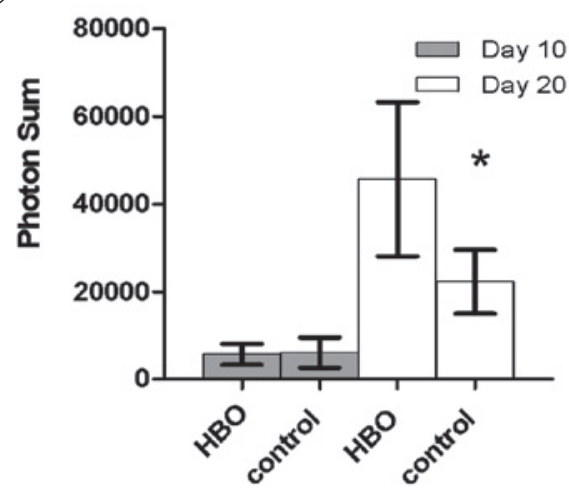

Figure 1. Hyperbaric oxygen (HBO) promoted the growth of intracranially transplanted GL261-Luc glioma cells in vivo. (A) In vivo bioluminescent imaging of tumors inoculated in HBO and control groups. (B) Photon light-emitting area and (C) photon sum of the HBO and control groups. Data are expressed as the mean \pm standard deviation. " $\mathrm{P}<0.05$ vs. HBO group.

A
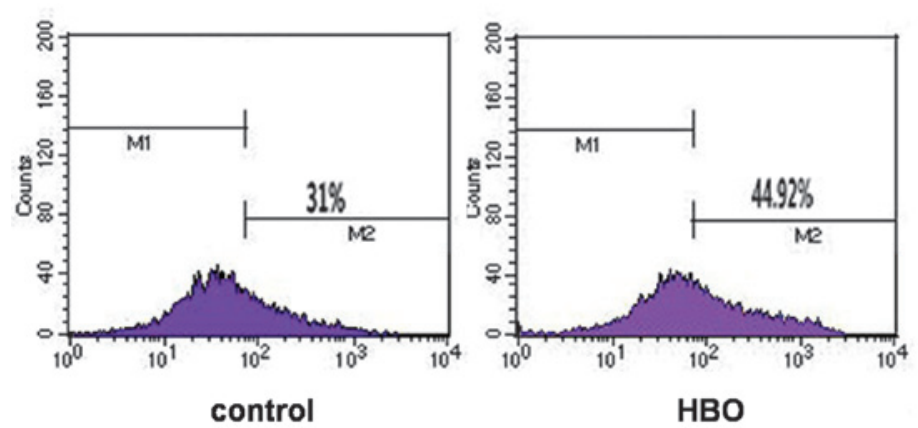

C

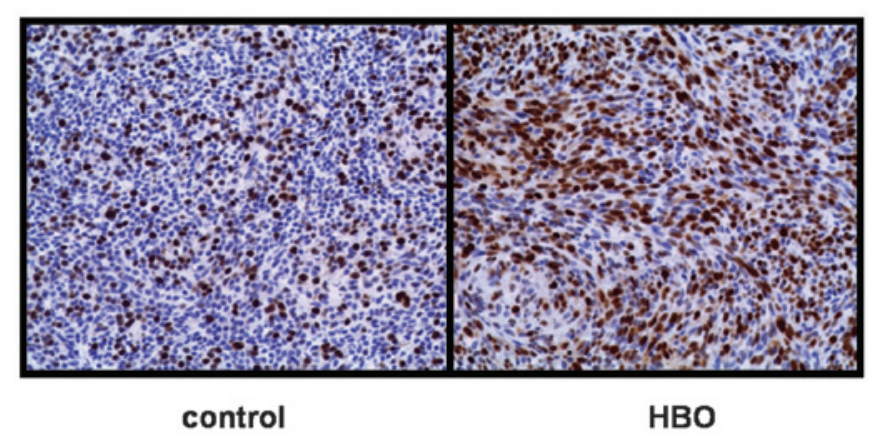

B

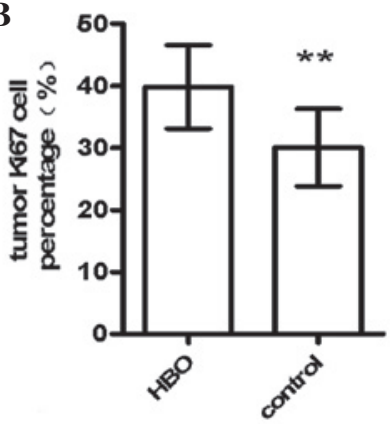

D

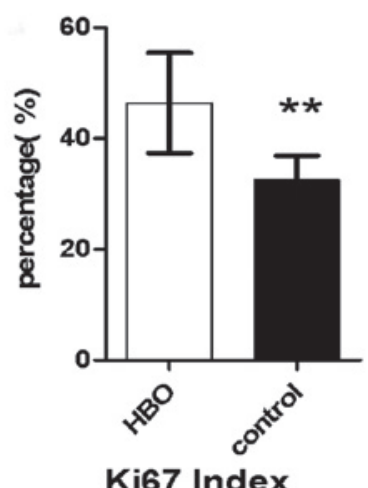

Figure 2. Hyperbaric oxygen (HBO) promoted the proliferation of glioma cells. (A) Flow cytometric analysis of tumors in the HBO and control groups. (B) Percentage of Ki67-positive glioma cells identified using flow cytometry in tumors inoculated in the HBO and control groups. (C) Images of Ki67 expression (stain, hematoxylin; magnification, x400) and (D) plot of the percentage of Ki67-positive glioma cells, identified using immunohistochemical analysis in tumors inoculated in the $\mathrm{HBO}$ and control groups. Data are expressed as the mean \pm standard deviation. ${ }^{* *} \mathrm{P}<0.01$ vs. $\mathrm{HBO}$ group.

indicated that the proportion of Ki67-positive glioma cells in the hyperbaric oxygen group was significantly increased compared with the control group (39.82 \pm 6.69 vs. $30.06 \pm 6.22$;
$\mathrm{P}=0.009$; Fig. 2A and B). Immunohistochemical analysis also indicated that the percentage of Ki67-positive cells was increased in the hyperbaric oxygen group compared with 

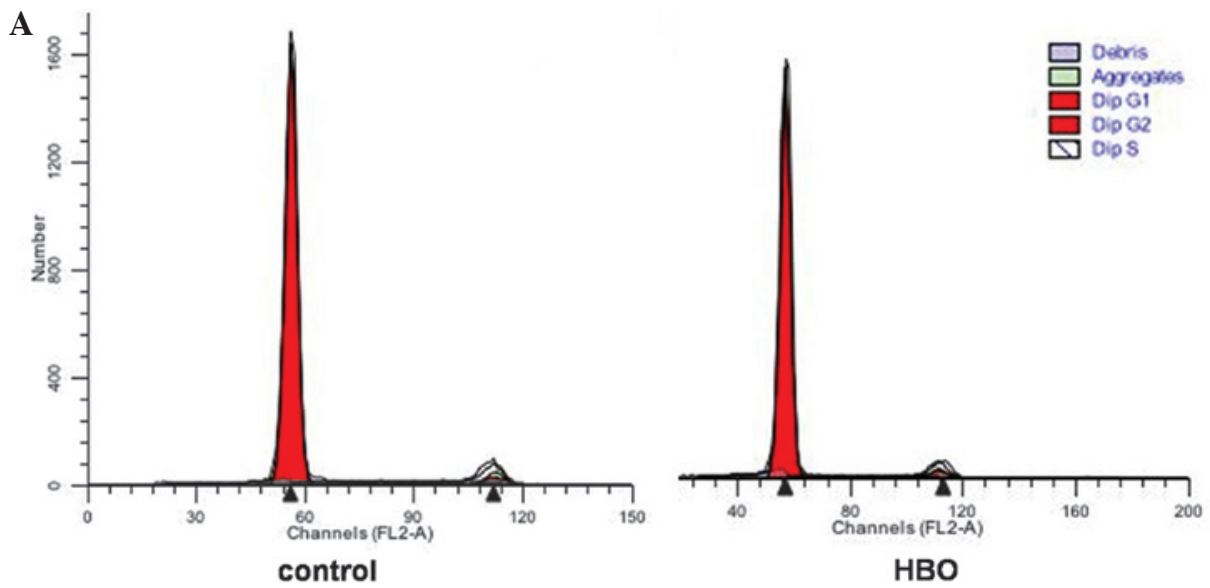

B

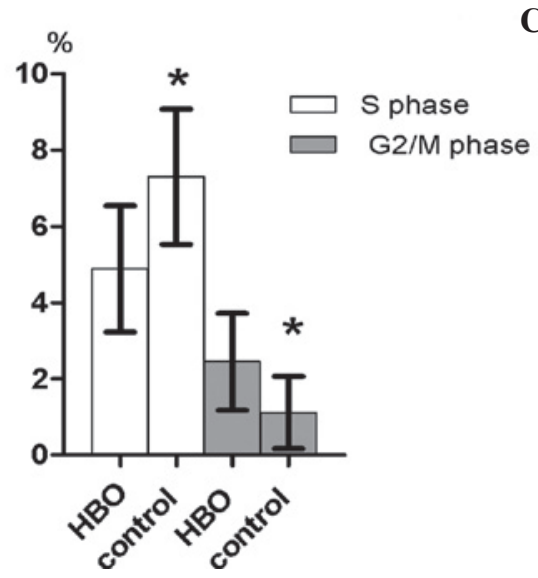

C \%

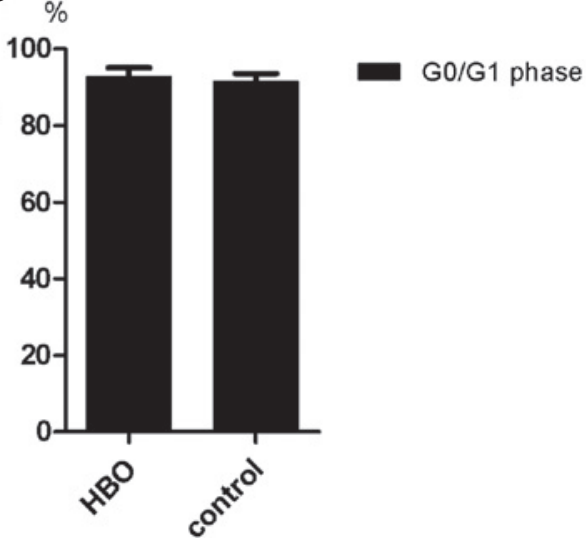

Figure 3. Hyperbaric oxygen (HBO) promoted glioma cells escaping cell cycle arrest. (A) Cell cycle analysis of tumors inoculated in the HBO and control groups. The percentages of glioma cells in the (B) $\mathrm{S}$ and $\mathrm{G}_{2} / \mathrm{M}$ phases, and $(\mathrm{C})$ in the $\mathrm{G}_{0} / \mathrm{G}_{1}$ phase of the cell cycle in tumors inoculated in $\mathrm{HBO}$ and control groups are demonstrated. Data are expressed as the mean \pm standard deviation. $\mathrm{P}<0.05$ vs. HBO group.

the control group $(46.33 \pm 9.03$ vs. $32.50 \pm 4.42 ; \mathrm{P}=0.007$ Fig. 2C and D).

Flow cytometric analysis of the cell cycle status demonstrated that the majority of glioma cells in the hyperbaric oxygen and control groups were in $\mathrm{G}_{0} / \mathrm{G}_{1}$ phase, while a small number of cells were in $\mathrm{G}_{2} / \mathrm{M}$ phase (Fig. $3 \mathrm{~A}$ and $\mathrm{B}$ ). The proportion of glioma cells in $\mathrm{S}$ phase of the hyperbaric oxygen group was significantly reduced compared with the control group (4.88 \pm 1.66 vs. $7.30 \pm 1.77 ; \mathrm{P}=0.014$; Fig. $3 \mathrm{C}$ ). In addition, the proportion of glioma cells in $\mathrm{G}_{2} / \mathrm{M}$ phase in the hyperbaric oxygen group was significantly increased compared with the control group $(2.45 \pm 1.27$ vs. $1.12 \pm 0.95 ; \mathrm{P}=0.033$; Fig. $3 \mathrm{C})$. However, no statistically significant difference was observed in the proportion of glioma cells in $G_{0} / G_{1}$ phase between the two groups (92.67 \pm 2.35 vs. $91.58 \pm 1.98 ; \mathrm{P}=0.334$; Fig. 3D).

Hyperbaric oxygen inhibited the apoptosis of glioma cells and promoted angiogenesis in transplanted glioma. Flow cytometric analysis indicated that the proportion of apoptotic glioma cells (Annexin V/PI double positive) in the hyperbaric oxygen group was significantly increased compared with the control group (13.75 \pm 8.84 vs. 8.27 $\pm 3.19 ; \mathrm{P}=0.023$; Fig. $4 \mathrm{~A}$ and $\mathrm{B})$. By contrast, the proportion of normal apoptotic brain cells in the hyperbaric oxygen group was significantly reduced compared with the control group (14.70 \pm 6.73 vs. $24.63 \pm 9.66$; $\mathrm{P}=0.032$; Fig. $4 \mathrm{~A}$ and $\mathrm{B}$ ). Immunohistochemical analysis also indicated that the number of TUNEL-positive glioma cells in the hyperbaric oxygen group was increased compared with the control group (Fig. 4C).

CD34 expression is associated with the angiogenesis capacity of vascular endothelial cells. Therefore, an increase in CD34 immunostaining was observed in the hyperbaric oxygen group compared with the control group (Fig. 5A), indicating that the angiogenesis capacity of the vascular endothelial cells of the transplanted glioma was increased by hyperbaric oxygen treatment.

The present study also compared the microvessel density (MVD) of glioma following hyperbaric oxygen treatment. The MVD in the hyperbaric oxygen group was significantly increased compared with the control group $(9.18 \pm 2.53$ vs. $19.80 \pm 3.33, \mathrm{P}=0.0003$; Fig. $5 \mathrm{~B}$ ), which further demonstrated that the angiogenesis capacity of vascular endothelial cells in the transplanted glioma was increased in the hyperbaric oxygen group.

\section{Discussion}

A number of previous studies have indicated that combining hyperbaric oxygen treatment with chemoradiotherapy may improve the prognosis for glioma patients and reduce complications $(13-15,22)$; however, the specific mechanism by which this occurs remains unknown. A previous study proposed that hyperbaric oxygen may induce apoptosis in tumor cells and decrease the vascular density in subcutaneous 
A
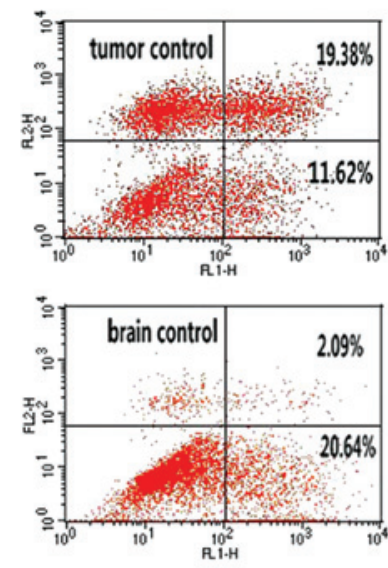

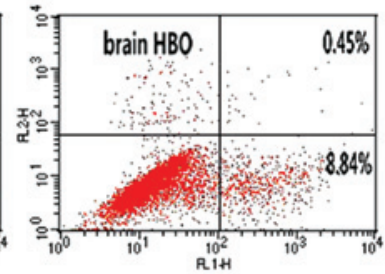

B
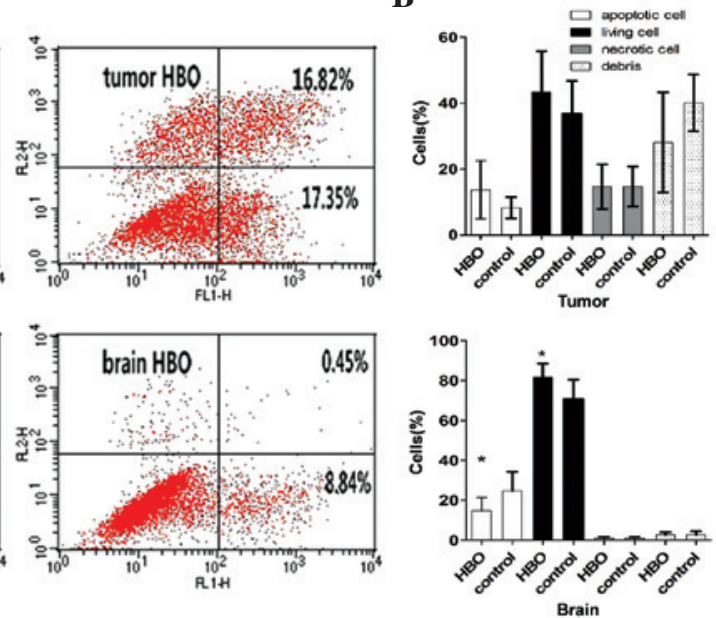

C

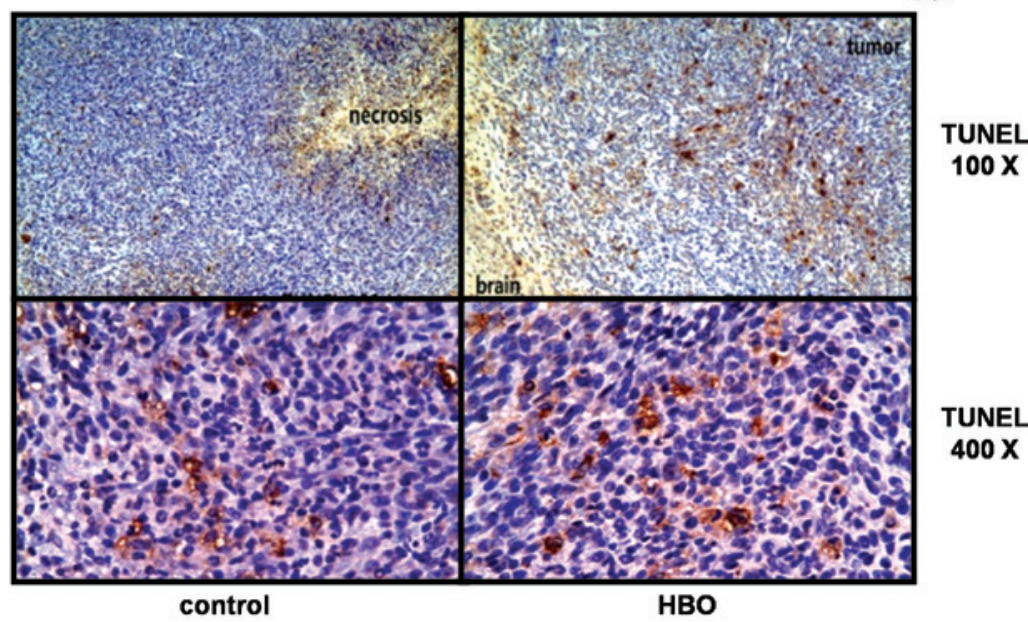

Figure 4. Hyperbaric oxygen (HBO) suppressed apoptosis in glioma cells. (A) Cell apoptosis of tumors inoculated in HBO and control groups, analyzed using flow cytometry. (B) Percentage of glioma cells in different survival status in the $\mathrm{HBO}$ and control groups. (C) Immunohistochemical analysis results of TUNEL staining in tumors inoculated in the HBO and control groups. Data are expressed as the mean \pm standard deviation. "P $\mathrm{P}<0.05$ vs. control group.

transplanted glioma tumors (18). Due to the blood-brain barrier, numerous therapies that have proven effective for treating subcutaneous transplanted gliomas, may not be effective in treating intracranial primary gliomas (19). Therefore, the present study produced an intracranial transplanted glioma model in congenic mice to investigate the direct effects of hyperbaric oxygen stimulation on glioma cells.

In vivo bioluminescent imaging (BLI) was applied in the present study for the $\mathrm{C} 57 \mathrm{BL} / 6 \mathrm{~J}$ mice tumorigenicity assay, in order to continuously and dynamically monitor the biological function of tumor cells in real-time, without affecting the physiological function of C57BL/6J mice $(23,24)$. A previous study indicated that hyperbaric oxygen increased the oxygen partial pressure of the intracranial tumor and this effect remained for $40 \mathrm{~min}$ following hyperbaric oxygen therapy (11). To avoid the interference of oxygen partial pressure, BLI was employed $12 \mathrm{~h}$ following hyperbaric oxygen therapy. In the present study, BLI indicated that the light-emitting area and the sum of emitted photons were increased in mice treated with hyperbaric oxygen, indicating that hyperbaric oxygen promoted growth of the intracranial glioma.

$\mathrm{Ki} 67$ is expressed in all phases of the cell cycle, with the exception of the $G_{0}$ phase, and therefore its expression is
A

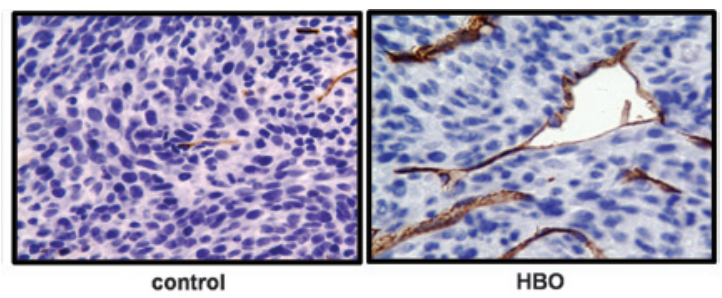

B

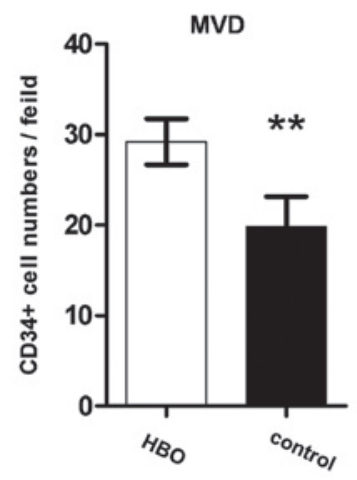

Figure 5. Hyperbaric oxygen (HBO) promoted angiogenesis of intracranially transplanted GL261-Luc glioma in vivo. (A) Immunohistochemical image of CD34 staining in tumors inoculated in the $\mathrm{HBO}$ and control groups (stain, hematoxylin; magnification, $x 400$ ). (B) Number of CD34+ cells per field in tumors of HBO and control groups. Data are expressed as the mean \pm standard deviation. ${ }^{* *} \mathrm{P}<0.01$ vs. $\mathrm{HBO}$ group. 
associated with cell proliferation. The results of immunohistochemical and flow cytometric analyses indicated that the expression of $\mathrm{Ki} 67$ in tumors repeatedly exposed to hyperbaric oxygen was increased compared with the control group. In contrast to the subcutaneous model, hyperbaric oxygen also increased the level of apoptosis in intracranial glioma cells in C57BL/6J mice. The proportion of TUNEL-positive cells was $\geq 60 \%$ in the control group, whereas the proportion of Ki67-positive cells was $\leq 10 \%$. In addition, in a previous study (15), for subcutaneous glioma cells, the proportion of apoptotic and proliferative cells was $\geq 60 \%$ and $\leq 10 \%$, respectively, whereas in the present study, the proportion of apoptotic cells in the intracranial glioma was $\leq 20 \%$, while the proportion of proliferative cells was $\geq 30 \%$. This difference may be due to the differences in the surroundings of the glioma cells. Therefore, the subcutaneous microenvironment may delay the growth of glioma cells. The increased proportion of apoptosis was associated with an increased capacity of self-renewal, which may have resulted from the glioma cells adapting to ambient pressure. Furthermore, the decreased proportion of proliferation may be associated with an adverse influence on the growth of glioma cells.

The present study also assessed the angiogenesis of tumor cells. Cells originating from glioma may participate in angiogenesis; glioma cells have been observed around blood vessels $(25,26)$, leading to the apoptosis of vascular cells and the destruction of vascular integrity, resulting in angiogenesis. The increased density and morphological change of capillaries is associated with an increased degree of malignancy and a poorer prognosis $(27,28)$. In the present study, the results demonstrated that increased MVD was associated with the proliferation of glioma cells, thus promoting angiogenesis; however, the opposite was observed in a subcutaneous model (18) and in breast cancer (29). The aforementioned observations indicate that the same intervention in different microenvironments may influence the growth of glioma cells differently (19). In addition, another previous study indicated that MVD was an effective prognostic factor, although no direct association was observed between MVD and therapeutic effect (30).

In conclusion, repeated exposure to hyperbaric oxygen promoted the proliferation and angiogenesis of intracranial glioma cells, inhibited apoptosis and prevented cell cycle arrest. Therefore, hyperbaric oxygen therapy may be a potentially effective therapeutic option and may improve the prognosis for patients with glioma.

\section{References}

1. Stupp R, Mason WP, van den Bent MJ, et al; European Organisation for Research and Treatment of Cancer Brain Tumor and Radiotherapy Groups; National Cancer Institute of Canada Clinical Trials Group: Radiotherapy plus concomitant and adjuvant temozolomide for glioblastoma. N Engl J Med 352: 987-996, 2005

2. Johnson DR and O'Neill BP: Glioblastoma survival in the United States before and during the temozolomide era. J Neurooncol 107: 359-364, 2012.

3. Sayegh ET, Kaur G, Bloch O and Parsa AT: Systematic review of protein biomarkers of invasive behavior in glioblastoma. Mol Neurobiol 49: 1212-1244, 2014.

4. Chamberlain MC: Neuro-oncology: A selected review of ASCO 2014 abstracts. CNS Oncology 3: 321-325, 2014.
5. Sathornsumetee S, Cao Y, Marcello JE, et al: Tumor angiogenic and hypoxic profiles predict radiographic response and survival in malignant astrocytoma patients treated with bevacizumab and irinotecan. J Clin Oncol 26: 271-278, 2008.

6. Jensen RL: Brain tumor hypoxia: Tumorigenesis, angiogenesis, imaging, pseudoprogression, and as a therapeutic target. J Neurooncol 92: 317-335, 2009.

7. Zhou Y, Zhou Y, Shingu T, et al: Metabolic alterations in highly tumorigenic glioblastoma cells: Preference for hypoxia and high dependency on glycolysis. J Biol Chem 286: 32843-32853, 2011.

8. Bar EE: Glioblastoma, cancer stem cells and hypoxia. Brain Pathol 21: 119-129, 2011

9. Persano L, Rampazzo E, Della Puppa A, Pistollato F and Basso G: The three-layer concentric model of glioblastoma: Cancer stem cells, microenvironmental regulation, and therapeutic implications. ScientificWorldJournal 11: 1829-1841, 2011

10. Brizel DM, Lin S, Johnson JL, Brooks J, Dewhirst MW and Piantadosi CA: The mechanisms by which hyperbaric oxygen and carbogen improve tumour oxygenation. Br J Cancer 72: 1120-1124, 1995.

11. Beppu T, Kamada K, Yoshida Y, Arai H, Ogasawara K and Ogawa A: Change of oxygen pressure in glioblastoma tissue under various conditions. J Neurooncol 58: 47-52, 2002.

12. Beppu T, Kamada K, Nakamura R, et al: A phase II study of radiotherapy after hyperbaric oxygenation combined with interferon- $\beta$ and nimustine hydrochloride to treat supratentorial malignant gliomas. J Neurooncol 61: 161-170, 2003.

13. Kohshi K, Kinoshita Y, Imada H, et al: Effects of radiotherapy after hyperbaric oxygenation on malignant gliomas. Br J Cancer 80: 236-241, 1999.

14. Ogawa K, Ishiuchi S, Inoue O, et al: Phase II trial of radiotherapy after hyperbaric oxygenation with multiagent chemotherapy (procarbazine, nimustine, and vincristine) for high-grade gliomas: Long-term results. Int J Radiat Oncol Biol Phys 82: 732-738, 2012 .

15. Kohshi K, Yamamoto H, Nakahara A, Katoh T and Takagi M: Fractionated stereotactic radiotherapy using gamma unit after hyperbaric oxygenation on recurrent high-grade gliomas. J Neurooncol 82: 297-303, 2007.

16. Sun S, Lee D, Lee NP, et al: Hyperoxia resensitizes chemoresistant human glioblastoma cells to temozolomide. J Neurooncol 109: 467-475, 2012.

17. Lu XY, Cao K, Li QY, Yuan ZC and Lu PS: The synergistic therapeutic effect of temozolomide and hyperbaric oxygen on glioma U251 cell lines is accompanied by alterations in vascular endothelial growth factor and multidrug resistance-associated protein-1 levels. J Int Med Res 40: 995-1004, 2012.

18. Stuhr LE, Raa A, Oyan AM, et al: Hyperoxia retards growth and induces apoptosis, changes in vascular density and gene expression in transplanted gliomas in nude rats. J Neurooncol 85: 191-202, 2007.

19. Biollaz G, Bernasconi L, Cretton C, et al: Site-specific anti-tumor immunity: Differences in DC function, TGF- $\beta$ production and numbers of intratumoral Foxp3+ Treg. Eur J Immunol 39: 1323-1333, 2009.

20. Conconi MT, Baiguera S, Guidolin D, et al: Effects of hyperbaric oxygen on proliferative and apoptotic activities and reactive oxygen species generation in mouse fibroblast 3T3/J2 cell line. J Investig Med 51: 227-232, 2003.

21. Milovanova TN, Bhopale VM, Sorokina EM, et al: Hyperbaric oxygen stimulates vasculogenic stem cell growth and differentiation in vivo. J Appl Physiol (1985) 106: 711-728, 2009.

22. Kohshi K, Kinoshita Y, Terashima H,Konda N, Yokota A and Soejima T: Radiotherapy after hyperbaric oxygenation for malignant gliomas: A pilot study. J Cancer Res Clin Oncol 122: 676-678, 1996.

23. Maes W, Deroose C, Reumers V, et al: In vivo bioluminescence imaging in an experimental mouse model for dendritic cell based immunotherapy against malignant glioma. J Neurooncol 91: 127-139, 2009.

24. Stafford P, Abdelwahab MG, Kim Y, Preul MC, Rho JM and Scheck AC: The ketogenic diet reverses gene expression patterns and reduces reactive oxygen species levels when used as an adjuvant therapy for glioma. Nutr Metab (Lond) 7: 74-74, 2010.

25. Calabrese C, Poppleton H, Kocak M, et al: A perivascular niche for brain tumor stem cells. Cancer Cell 11: 69-82, 2007.

26. Zagzag D, Amirnovin R, Greco MA, et al: Vascular apoptosis and involution in gliomas precede neovascularization: A novel concept for glioma growth and angiogenesis. Lab Invest 80: $837-849,2000$. 
27. Deb P,Boruah D and Dutta V: Morphometric study of microvessels in primary CNS tumors and its correlation with tumor types and grade. Microvasc Res 84: 34-43, 2012.

28. Leon SP, Folkerth RD and Black PM: Microvessel density is a prognostic indicator for patients with astroglial brain tumors. Cancer 77: 362-372, 1996.

29. Moen I, Jevne C, Wang J, et al: Gene expression in tumor cells and stroma in dsRed 4T1 tumors in eGFP-expressing mice with and without enhanced oxygenation. BMC Cancer 12: 21-21, 2012.
30. Preusser M, Heinzl H, Gelpi E, et al; European Organization for Research and Treatment of Cancer Brain Tumor Group: Histopathologic assessment of hot-spot microvessel density and vascular patterns in glioblastoma: Poor observer agreement limits clinical utility as prognostic factors: A translational research project of the European Organization for Research and Treatment of Cancer Brain Tumor Group. Cancer 107: 162-170, 2006. 